\title{
The 6-metre Altazimuth Telescope
}

\author{
N. N. MIHEL'SON \\ Pulkovo Observatory
}

(Dr. B. K. Ioannisiani was unable to give his scheduled paper on this subject, but Dr. N. N. Mihel'son kindly agreed to answer questions in its place.)

\section{DISCUSSION}

R. F. NIELSEN : Would you be so kind as to comment upon the drive system for the 6-metre telescope? N. N. MiHEL'son: The telescope is now at the stage of mounting in the observatory. The fork has been mounted in the dome, and the tube in the workshops, and we hope that in the winter they will be assembled together. The drive system has been mounted and tested in the workshops, and we hope that within the next year it will be tested together with the telescope. The system includes a digital computer, with 27-bit words, using 20 bits for the readout system, for azimuth and zenith distance. The computer will use right ascension, declination, and sidereal time, together with fully automatic correction for temperature and atmospheric pressure, to calculate the refraction correction. The tube deflection will be measured and a second program will compensate for this. From right ascension, declination, and sidereal time, the computer will calculate azimuth, zenith distance, and parallactic angle, and also the rates of change of these angles. Every tenth second these angles will be measured from the telescope, and the difference from the calculated values will be fed back to the telescope. Between these $10 \mathrm{sec}$, the data will be interpolated every $1 / 8$ th sec to control the speeds of the drive motors. The telescope has two main gears, a worm gear and a spur gear, and only one motor will work both.

E. W. DENNISON: What kind of motors?

N. N. MineL'Son: These are variable speed motors, but I do not know the English word.

J. L. LOWRANCE: What is the material of the primary mirror, and what figuring do you expect?

N. N. Minel'son: It is a super-pyrex, similar to the Hale Telescope. I must tell you also about the photoguider. The guider tube, $700 \mathrm{~mm}$ in diameter, will be parallel to the main telescope tube. It will be a Babcock-type guider, working on the main motors of the telescope. There will be further photoguiders at the prime and Nasmyth foci, working only on the plate cassette or on a plane-parallel glass plate in front of the spectrograph slit. We hope also to have a television guider. Field rotation will be compensated by the digital computer and also by two photoguiders.

R. E. NATHER: May I offer a word of warning about this system? We tried the same arrangement on the 107-inch at McDonald, using a separate guider telescope mounted on the main tube. We found that differential fiexure between the large telescope and the small one was so great that we had to abandon this system. I am worried that you might be in the same trouble.

N. N. Minel'son: We have calculated the flexure, and we have photoguiders in the focal plane, in addition to that on the guider telescope. Thus we have two stages of autoguidance.

G. VAN HERK: How far out of the meridian will you be able to use the telescope?

N. N. MiHeL'son: There are dead zones within $5^{\circ}$ of the zenith and also within $10^{\circ}$ of the horizon.

W. A. SHER WOOD: What practical work, not calculations, has been done on field rotation?

N. N. MIHEL'son: It has been investigated on a simulator.

SCHLOSSER: Do you intend to write a special compiler for the computer, so as to facilitate observations by writing simply "observation-programs"?

N. N. MiHel'son: No, this is a special-purpose computer. The program is held in the core memory and cannot be changed.

A. T. PURGathofer: What is the linear expansion-coefficient for your super-pyrex? 
N. N. Minel'son: I believe it is in the range 25 to $35 \times 10^{-7}$, similar to the Hale Telescope.

P. Gillingham: What is the upper frequency limit with which you hope to make corrections with the local autoguiders?

N. N. MUHEL'son: I am sorry, I do not know.

W. LILLER: When will the telescope be in operation?

N. N. MIHEL'sON : A very difficult question! We hope that the mirror polishing will be complete by the time the other systems are all working. A concrete dummy will replace the main mirror during preliminary tests.

\section{REFERENCES}

Ioannisiani, B. K., 1970. The Development of the 6-metre Reflecting Telescope, Optiko-Mehaničeskaja Promyšlennost', 37, 37.

Besekerskij, V. A. and Neplohov, E. M., 1970. Use of the principle of invariance in the control system of an optical altazimuth telescope, in The Theory of Invariance in Automatic Systems, Moscow, p. $319,1970$. 